\title{
OPTIMAL BUY/SELL RULES FOR CORRELATED RANDOM WALKS
}

\author{
PIETER ALLAART *** AND \\ MICHAEL MONTICINO, ${ }^{* * * *}$ University of North Texas
}

\begin{abstract}
Correlated random walks provide an elementary model for processes that exhibit directional reinforcement behavior. This paper develops optimal multiple stopping strategies—buy/sell rules-for correlated random walks. The work extends previous results given in Allaart and Monticino (2001) by considering random step sizes and allowing possibly negative reinforcement of the walk's current direction. The optimal strategies fall into two general classes-cases where conservative buy-and-hold type strategies are optimal and cases for which it is optimal to follow aggressive trading strategies of successively buying and selling the commodity depending on whether the price goes up or down. Simulation examples are given based on a stock index fund to illustrate the variation in return possible using the theoretically optimal stop rules compared to simpler buy-and-hold strategies.
\end{abstract}

Keywords: Correlated random walk; multiple stopping; buy/sell strategy

2000 Mathematics Subject Classification: Primary 60G40; 60G50

\section{Introduction}

This paper develops optimal multiple stopping strategies_-buy/sell rules_for commodities whose prices follow a generalized class of correlated random walks. The work extends previous results given in Allaart and Monticino (2001) by considering random step sizes and allowing for possibly negative reinforcement of a walk's current direction. Interestingly, the optimal strategies fall into two general classes - cases where a conservative buy-and-hold strategy is optimal and cases for which it is optimal to follow a very aggressive trading strategy of successively buying and selling the commodity depending on whether the price goes up or down.

Define a random walk with correlation (RWC), $\left\{S_{n}\right\}_{n \geq 0}$, by $S_{0} \equiv s_{0} \in \mathbb{R}$ and $S_{n}=S_{0}+$ $X_{1}+\cdots+X_{n}$ for $n \geq 1$, where the increments $X_{1}, X_{2}, \ldots$ form a Markov sequence in the following way. Let $Y_{u}$ and $Y_{d}$ be real-valued random variables with finite expectations. For each $n \in \mathbb{N}, \mathcal{L}\left(X_{n+1} \mid X_{n} \geq 0\right)=\mathcal{L}\left(Y_{u}\right)$ and $\mathcal{L}\left(X_{n+1} \mid X_{n}<0\right)=\mathcal{L}\left(Y_{d}\right)$, where $\mathcal{L}$ denotes the probability law. Let $p=\mathrm{P}\left(Y_{u} \geq 0\right)$ and $q=\mathrm{P}\left(Y_{d}<0\right)$. (Assume that $0<p, q<1$ to avoid uninteresting cases.) So, if the price goes up at time $n$, it will go up (or stay equal) at time $n+1$ with probability $p$ and will go down with probability $1-p$. The distribution of the price change at time $n+1$ after a price increase at time $n$ is $\mathcal{L}\left(Y_{u}\right)$. Similarly, if the price goes down at time $n$, it will go down at time $n+1$ with probability $q$ and will go up or stay the

Received 1 November 2005; revision received 3 January 2008.

* Postal address: Mathematics Department, University of North Texas, PO Box 311430, Denton, TX 76203-1430, USA.

** Email address: allaart@unt.edu

*** Email address: monticino@unt.edu 
same with probability $1-q$. The distribution of the price change at time $n+1$ given a price decrease at time $n$ is $\mathcal{L}\left(Y_{d}\right)$.

The objective of this paper is to determine a sequence of buying and selling times that maximize the investor's expected return from trading a commodity whose price follows the walk $S_{n}$, given an investment time horizon $N$ and transaction $\operatorname{cost} c \geq 0$. That is, the goal is to find stopping times

$$
0 \leq \tau_{1}<\tau_{2}<\cdots<\tau_{2 m} \leq N
$$

such that if the commodity is bought at times $\tau_{2 j-1}$ and sold at times $\tau_{2 j}$ then the expected value of the total return

$$
R(N)=\sum_{j=1}^{m}\left(S_{\tau_{2 j}}-S_{\tau_{2 j-1}}\right)-2 m c
$$

is maximized, where $m$ is possibly random. Note that the investor incurs a transaction cost for each trade whether it is a buy or a sell. Since the investor is required to sell the commodity by time $N$ if it is ever bought, each buy is paired with a sell.

Optimal trading strategies are developed for four basic cases of commodity price behavior. The first is trivial: the price process is a supermartingale and the obvious optimal strategy is never to buy. On the other hand, when the price process is a submartingale, it is optimal to hold onto the commodity until the time horizon once the commodity is purchased. It is just a question of whether to buy given the investment time horizon. The third case considered is when the price is expected to decrease on the next step if the price went up on the previous stage, and the price is expected to increase if the price went down on the previous stage. The form of the optimal strategy depends on the transaction costs for this case. Either the commodity is bought and sold a single time according to buy and sell signals or, if costs are low enough, it is optimal to successively buy and sell according to the familiar investment axiom of 'buying on the dips and selling on the peaks'. The final case considered reverses the commodity behavior and optimal strategies of the third case. Optimal strategies for all cases are given in the next section.

The random walk model considered here is an immediate extension of the correlated random walks introduced in Goldstein (1951). Basic properties of correlated random walks such as transition probabilities and first passage times have been examined in a number of papers. For instance, Seth (1963) gave return probabilities and first-passage time distributions for symmetric correlated random walks. Jain (1971) generalized these results to the nonsymmetric case. Renshaw and Henderson (1981) presented occupation probabilities and a diffusion approximation. Gillis (1955) developed a $d$-dimensional version, and conjectured it to be transient for all $d \geq 3$. Gillis' conjecture was proved in Iossif (1986) and then for more general correlated random walks in Chen and Renshaw (1994). Other results on correlated random walks and boundary problems include Proudfoot and Lampard (1972), Jain (1973), Mukherjea and Steele (1987), Zhang (1992), and Böhm (2000).

Gambler ruin type problems for correlated random walks are examined in Mohan (1955) and Mukherjea and Steele (1986). Optimal buy/sell strategies are developed for a more general class of processes called directionally reinforced random walks in Allaart and Monticino (2001). Results given there are extended here by allowing random step sizes and accounting for negative reinforcement addressed by the 'buy on the dips and sell on the peaks' strategy. Allaart (2004) examined the single stop problem of when to sell a commodity whose price follows a correlated random walk in order to maximize the discounted return. 
Other applications using correlated random walks include Goldstein (1951), where certain physical diffusion processes were modeled, and Henderson and Renshaw (1980) in which tree root growth was examined. Renshaw and Henderson (1981) studied the behavior of a certain kind of pinball machine. Mauldin et al. (1996) used the more general directionally reinforced random walks as an elementary model of ocean surface waves. A comprehensive list of references for correlated random walks is given in Chen and Renshaw (1994).

The problem explored here is motivated by the popular notion among proponents of stock market technical analysis that movements in security prices are not memoryless. In particular, price changes - up or down - from one day to the next affect succeeding day changes. Various assertions are given to justify this idea of price momentum, such as fundamental information about a company ripples out from insiders to investment professionals to individual investors. As this happens, the theory goes that the stock price is pushed ever higher if the information is favorable, or lower if the news is bad. Of course, it is far from agreed that the market consistently exhibits any behavior other than a random walk about an underlying trend (see, for instance, Malkiel (1999)). Regardless of one's belief in this postulated market phenomena, very little seems to be known about optimal stopping for processes exhibiting momentum. The intention here is not to take a stand on the existence of price momentum in the market, but to gain insight into how an investor might take advantage of momentum, if present, by examining a simple model of such processes. In Section 3 we illustrate the strategies developed through the behavior of a stock index fund. Using a model based on the price history of the fund, simulation results are given which indicate the variation in return possible from using theoretically optimal stop rules. The simulations also compare the performance of optimal stop rules to simpler buy-and-hold strategies.

\section{Optimal buy/sell strategies}

In this section we present optimal buy/sell strategies. As mentioned above, there are four main cases that determine the form of the optimal strategy. Some necessary notation is developed first.

Let $E^{+}:=\mathrm{E}\left[Y_{u}\right]$ and $E^{-}:=\mathrm{E}\left[Y_{d}\right]$. Define the total correlation by $r:=p+q-1$ and the drift by

$$
\delta:=\frac{(1-q) E^{+}+(1-p) E^{-}}{1-r} .
$$

Recall that each purchase of the commodity is paired with a sell. So, without loss of generality, assume that a single transaction $\operatorname{cost} C$ representing total buying and selling costs is (only) assessed when the commodity is bought.

For $j \in \mathbb{N}$, let $V_{\mathrm{H}}^{+}(j)$ denote the optimal expected additional net gain when there are $j$ time periods remaining, when the last increment was nonnegative, and when after seeing that increment, a decision was made resulting in the commodity now being held. Let $V_{\mathrm{F}}^{+}(j)$ denote the same, except that after the last decision moment, the commodity is not being held ('free' state). Similarly, define $V_{\mathrm{H}}^{-}(j)$ and $V_{\mathrm{F}}^{-}(j)$, replacing 'nonnegative' with 'negative'. For convenience, define $V_{\mathrm{H}}^{+}(0)=V_{\mathrm{H}}^{-}(0)=V_{\mathrm{F}}^{+}(0)=V_{\mathrm{F}}^{-}(0)=0$. The following recursive 
relationships hold:

$$
\begin{aligned}
& V_{\mathrm{H}}^{+}(j+1)=E^{+}+p \max \left\{V_{\mathrm{H}}^{+}(j), V_{\mathrm{F}}^{+}(j)\right\}+(1-p) \max \left\{V_{\mathrm{H}}^{-}(j), V_{\mathrm{F}}^{-}(j)\right\}, \\
& V_{\mathrm{F}}^{+}(j+1)=p \max \left\{V_{\mathrm{H}}^{+}(j)-C, V_{\mathrm{F}}^{+}(j)\right\}+(1-p) \max \left\{V_{\mathrm{H}}^{-}(j)-C, V_{\mathrm{F}}^{-}(j)\right\}, \\
& V_{\mathrm{H}}^{-}(j+1)=E^{-}+(1-q) \max \left\{V_{\mathrm{H}}^{+}(j), V_{\mathrm{F}}^{+}(j)\right\}+q \max \left\{V_{\mathrm{H}}^{-}(j), V_{\mathrm{F}}^{-}(j)\right\}, \\
& V_{\mathrm{F}}^{-}(j+1)=(1-q) \max \left\{V_{\mathrm{H}}^{+}(j)-C, V_{\mathrm{F}}^{+}(j)\right\}+q \max \left\{V_{\mathrm{H}}^{-}(j)-C, V_{\mathrm{F}}^{-}(j)\right\} .
\end{aligned}
$$

Lemma 1, below, collects some elementary properties of the functions defined above. The straightforward proofs are omitted.

Lemma 1. (a) $V_{\mathrm{H}}^{+}(j), V_{\mathrm{F}}^{+}(j), V_{\mathrm{H}}^{-}(j)$, and $V_{\mathrm{F}}^{-}(j)$ are all nondecreasing in $j$.

(b) $V_{\mathrm{F}}^{+}(j) \geq 0$ and $V_{\mathrm{F}}^{-}(j) \geq 0$ for all $j$.

(c) If $E^{+} \geq 0$ then $V_{\mathrm{H}}^{+}(j) \geq V_{\mathrm{F}}^{+}(j)$ for all $j$. If $E^{-} \geq 0$ then $V_{\mathrm{H}}^{-}(j) \geq V_{\mathrm{F}}^{-}(j)$ for all $j$.

(d) If $E^{+} \leq 0$ then $V_{\mathrm{H}}^{+}(j)-C \leq V_{\mathrm{F}}^{+}(j)$ for all $j$. If $E^{-} \leq 0$ then $V_{\mathrm{H}}^{-}(j)-C \leq V_{\mathrm{F}}^{-}(j)$ for all $j$.

(e) If $E^{+} \geq E^{-}$then $V_{\mathrm{H}}^{+}(j) \geq V_{\mathrm{H}}^{-}(j)$ and $V_{\mathrm{F}}^{+}(j) \geq V_{\mathrm{F}}^{-}(j)$ for all $j$. If $E^{+} \leq E^{-}$, the reverse inequalities hold.

Next, define the differences

$$
\begin{array}{ll}
D^{+}(j):=V_{\mathrm{H}}^{+}(j)-V_{\mathrm{F}}^{+}(j), & D^{-}(j):=V_{\mathrm{H}}^{-}(j)-V_{\mathrm{F}}^{-}(j), \\
D_{\mathrm{H}}(j):=V_{\mathrm{H}}^{+}(j)-V_{\mathrm{H}}^{-}(j), & D_{\mathrm{F}}(j):=V_{\mathrm{F}}^{+}(j)-V_{\mathrm{F}}^{-}(j) .
\end{array}
$$

Observe that with $j$ time periods remaining, it is optimal to buy after an up-step if and only if $D^{+}(j) \geq c$ and it is optimal to buy after a down-step if and only if $D^{-}(j) \geq c$.

Lemma 2. (a) If $E^{+} \geq E^{-}$then $D^{+}(j) \geq D^{-}(j)$ for all $j \geq 0$.

(b) If $E^{+} \leq E^{-}$then $D^{+}(j) \leq D^{-}(j)$ for all $j \geq 0$.

Proof. Assume that $E^{+} \geq E^{-}$. For $j \geq 0$, define

$$
\begin{aligned}
\Delta(j)= & \max \left\{V_{\mathrm{H}}^{+}(j), V_{\mathrm{F}}^{+}(j)\right\}-\max \left\{V_{\mathrm{H}}^{-}(j), V_{\mathrm{F}}^{-}(j)\right\} \\
& -\max \left\{V_{\mathrm{H}}^{+}(j)-C, V_{\mathrm{F}}^{+}(j)\right\}+\max \left\{V_{\mathrm{H}}^{-}(j)-C, V_{\mathrm{F}}^{-}(j)\right\} .
\end{aligned}
$$

It will be shown inductively that, for all $j \in \mathbb{N}$,

$$
D^{+}(j) \geq D^{-}(j), \quad \Delta(j-1) \geq 0 .
$$

Since $D^{+}(1)-D^{-}(1)=E^{+}-E^{-}$and $\Delta(0)=0$, (5) holds for $j=1$. Assume that it holds for $j=k$. Then, by (1)-(4),

$$
\begin{aligned}
D^{+}(k+1)-D^{-}(k+1) & =D_{\mathrm{H}}(k+1)-D_{\mathrm{F}}(k+1) \\
& =E^{+}-E^{-}+r \Delta(k) .
\end{aligned}
$$

If $V_{\mathrm{H}}^{-}(k)-C \geq V_{\mathrm{F}}^{-}(k)$ then $V_{\mathrm{H}}^{+}(k)-C \geq V_{\mathrm{F}}^{+}(k)$ by the induction hypothesis and $\Delta(k)=0$. Likewise, if $V_{\mathrm{H}}^{+}(k) \leq V_{\mathrm{F}}^{+}(k)$ then $V_{\mathrm{H}}^{-}(k) \leq V_{\mathrm{F}}^{-}(k)$ and again $\Delta(k)=0$. In both cases (5) clearly holds for $j=k+1$. Assume, therefore, that

$$
V_{\mathrm{H}}^{-}(k)-C<V_{\mathrm{F}}^{-}(k) \text { and } \quad V_{\mathrm{H}}^{+}(k)>V_{\mathrm{F}}^{+}(k) \text {. }
$$


Then

$$
\Delta(k)=\min \left\{D^{+}(k), C\right\}-\max \left\{D^{-}(k), 0\right\} .
$$

This, along with the induction hypothesis and (7), implies that $\Delta(k) \geq 0$. If $r \geq 0$, this immediately yields $D^{+}(k+1) \geq D^{-}(k+1)$. If $r<0$ then, by (8) and the induction hypothesis,

$$
\Delta(k) \leq D^{+}(k)-D^{-}(k)=E^{+}-E^{-}+r \Delta(k-1) \leq E^{+}-E^{-} .
$$

Since $r \geq-1$, substituting (9) into (6) yields $D^{+}(k+1) \geq D^{-}(k+1)$. This proves part (a). The proof of part (b) is analogous.

Theorem 1. If it is optimal to buy after an up-step with $j$ time periods remaining then it is optimal to buy after an up-step with $j+1$ time periods remaining. The analogous statement holds for buying after a down-step.

Proof. Suppose that $D^{+}(j) \geq C$. By Lemma $1(\mathrm{~d})$, this implies that $E^{+} \geq 0$. Thus, if $V_{\mathrm{H}}^{-}(j)-C \geq V_{\mathrm{F}}^{-}(j)$ then (1) and (2) immediately yield $D^{+}(j+1) \geq C$. Suppose, therefore, that $V_{\mathrm{H}}^{-}(j)-C<V_{\mathrm{F}}^{-}(j)$. By Lemma 2 , this implies that $E^{+} \geq E^{-}$and, hence, by Lemma 1(e), $V_{\mathrm{F}}^{+}(j) \geq V_{\mathrm{F}}^{-}(j)$. Now $V_{\mathrm{H}}^{+}(j+1) \geq V_{\mathrm{H}}^{+}(j)$ and

$$
\begin{aligned}
V_{\mathrm{F}}^{+}(j+1) & =p\left\{V_{\mathrm{H}}^{+}(j)-C\right\}+(1-p) V_{\mathrm{F}}^{-}(j) \\
& \leq p\left\{V_{\mathrm{H}}^{+}(j)-C\right\}+(1-p) V_{\mathrm{F}}^{+}(j),
\end{aligned}
$$

so that

$$
D^{+}(j+1) \geq(1-p)\left\{V_{\mathrm{H}}^{+}(j)-V_{\mathrm{F}}^{+}(j)\right\}+p C \geq C .
$$

This completes the proof for buying after an up-step. The proof for buying after a down-step is similar.

The optimal buy/sell strategies are as follows.

Case I: $E^{+} \leq 0$ and $E^{-} \leq 0$. The price process, $S_{n}$, is a supermartingale in this case. Thus, the optimal strategy is not to buy the commodity at all.

Case II: $E^{+}>0$ and $E^{-}>0$. In this case the price process is a submartingale. Hence, once the commodity is bought, it is optimal to hold it until the time horizon. The question is whether to buy the commodity. Intuitively, the commodity should be bought only if there is enough time between purchase and the time horizon for the positive drift of the walk to generate an expected gain greater than the transaction cost.

In this case (1) and (3) simplify to

$$
\begin{aligned}
& V_{\mathrm{H}}^{+}(j+1)=E^{+}+p V_{\mathrm{H}}^{+}(j)+(1-p) V_{\mathrm{H}}^{-}(j), \\
& V_{\mathrm{H}}^{-}(j+1)=E^{-}+(1-q) V_{\mathrm{H}}^{+}(j)+q V_{\mathrm{H}}^{-}(j) .
\end{aligned}
$$

Hence,

$$
D_{\mathrm{H}}(j+1)=r D_{\mathrm{H}}(j)+\left(E^{+}-E^{-}\right), \quad j \geq 0 .
$$

Since $D_{\mathrm{H}}(0)=0$, it follows that

$$
D_{\mathrm{H}}(j)=\frac{E^{+}-E^{-}}{1-r}\left(1-r^{j}\right), \quad j \geq 0
$$


Substituting $V_{\mathrm{H}}^{-}(j)=V_{\mathrm{H}}^{+}(j)-D_{\mathrm{H}}(j)$ into (10) and iterating gives

$$
V_{\mathrm{H}}^{+}(j)=\sum_{k=1}^{j}\left(E^{+}-(1-p) D_{\mathrm{H}}(k-1)\right), \quad j \in \mathbb{N} .
$$

Thus, after some manipulations,

$$
V_{\mathrm{H}}^{+}(j)=\delta j+\frac{(1-p)\left(E^{+}-E^{-}\right)}{(1-r)^{2}}\left(1-r^{j}\right), \quad j \in \mathbb{N},
$$

and

$$
V_{\mathrm{H}}^{-}(j)=\delta j-\frac{(1-q)\left(E^{+}-E^{-}\right)}{(1-r)^{2}}\left(1-r^{j}\right), \quad j \in \mathbb{N} .
$$

There are two subcases.

Subcase II $(a)$ : $E^{+} \geq E^{-}$. Let $j^{+}$be the smallest $j$ for which $D^{+}(j) \geq C$, and let $j^{-}$be the smallest $j$ for which $D^{-}(j) \geq C$. Then $j^{+} \leq j^{-}$by Lemma 2 , and an easy induction argument shows that $V_{\mathrm{F}}^{+}(j)=V_{\mathrm{F}}^{-}(j)=0$ for all $j \leq j^{+}$. Thus, $j^{+}$is the smallest $j$ such that $V_{\mathrm{H}}^{+}(j) \geq C$. Since $\delta>0$, such a $j$ exists by (12).

If $V_{\mathrm{H}}^{-}\left(j^{+}\right) \geq C$ then $j^{-}=j^{+}$. Otherwise, let $j^{+} \leq j<j^{-}$. Then

$$
V_{\mathrm{F}}^{-}(j+1)=(1-q)\left\{V_{\mathrm{H}}^{+}(j)-C\right\}+q V_{\mathrm{F}}^{-}(j),
$$

so, by (3),

$$
D^{-}(j+1)=q D^{-}(j)+E^{-}+(1-q) C .
$$

Since

$$
D^{-}\left(j^{+}\right)=V_{\mathrm{H}}^{-}\left(j^{+}\right)-V_{\mathrm{F}}^{-}\left(j^{+}\right)=V_{\mathrm{H}}^{-}\left(j^{+}\right),
$$

it follows that, for all $j^{+}<j \leq j^{-}$,

$$
D^{-}(j)=\left\{V_{\mathrm{H}}^{-}\left(j^{+}\right)-b^{-}\right\} q^{j-j^{+}}+b^{-}, \quad b^{-}:=\frac{E^{-}}{1-q}+C .
$$

Hence, $j^{-}$is the smallest $j \geq j^{+}$such that $\left\{V_{\mathrm{H}}^{-}\left(j^{+}\right)-b^{-}\right\} q^{j-j^{+}}+b^{-} \geq C$. Since $b^{-}>C$, $j^{-}$is finite.

In summary, to determine whether to buy the commodity, first find $j^{+}$, the smallest $j$ such that $V_{\mathrm{H}}^{+}(j) \geq C$, using (12). Compute $V_{\mathrm{H}}^{-}\left(j^{+}\right)$using (13). Then use (14) to find $j^{-}$, the smallest $j \geq j^{+}$such that $D^{-}(j) \geq C$. Now, with $j$ time periods remaining, it is optimal to buy after an up-step if and only if $j \geq j^{+}$and it is optimal to buy after a down-step if and only if $j \geq j^{-}$. Again, the commodity is held until the time horizon if it is purchased.

Subcase II (b): $E^{+}<E^{-}$. Here the order of $j^{+}$and $j^{-}$is reversed. First, find $j^{-}$, the smallest $j$ such that $V_{\mathrm{H}}^{-}(j) \geq C$, using (13). Compute $V_{\mathrm{H}}^{+}\left(j^{-}\right)$using (12). Then use the formula

$$
D^{+}(j)=\left\{V_{\mathrm{H}}^{+}\left(j^{-}\right)-b^{+}\right\} p^{j-j^{-}}+b^{+}, \quad b^{+}:=\frac{E^{+}}{1-p}+C,
$$

to find $j^{+}$, the smallest $j \geq j^{-}$such that $D^{+}(j) \geq C$. Finally, use $j^{+}$and $j^{-}$as in Subcase II(a). 
Case III: $E^{+} \leq 0, E^{-}>0$. This case and the next are the most interesting as the optimal strategies may involve multiple trades in and out of the commodity. Some additional definitions are needed before specifying the optimal strategies.

Define

$$
j_{s}:=\inf \left\{j \geq 1: 1-q^{j} \geq \frac{(1-q)\left|E^{+}\right|}{(1-p) E^{-}}\right\},
$$

where the infimum of an empty set is taken to be $\infty$. Let

$$
f^{-}(j):= \begin{cases}\frac{1-q^{j}}{1-q} E^{-}, & j \leq j_{s}+1, \\ \left(j-j_{s}-1\right) \delta+r\left(q^{j_{s}} E^{-}-\delta\right) \frac{1-r^{j-j_{s}-1}}{1-r}+\frac{1-q^{j_{s}+1}}{1-q} E^{-}, & j>j_{s}+1,\end{cases}
$$

and define

$$
j_{b}:=\inf \left\{j \geq 1: f^{-}(j) \geq C\right\} .
$$

It is not difficult to see that $j_{s}$ is finite if and only if $\delta>0$. Similarly, $j_{b}$ is finite if and only if $\delta>0$ or $E^{-}>C(1-q)$.

Theorem 2. If $E^{+} \leq 0$ and $E^{-}>0$ then the optimal strategy is

(i) never sell after a down-step;

(ii) never buy after an up-step;

(iii) buy after a down-step if and only if there are at least $j_{b}$ time periods remaining;

(iv) sell after an up-step if and only if $\left|E^{+}\right| \geq C(1-p)$ or the number of remaining time periods is at most $\min \left(j_{b}, j_{s}\right)$.

Proof. Parts (i) and (ii) of the optimal strategy follow directly from Lemma 1(c) and Lemma 1(d), respectively. To prove parts (iii) and (iv), let $j^{*}$ be the smallest value of $j$ (possibly infinite) such that $D^{-}(j) \geq C$. Since $V_{\mathrm{F}}^{+}(j)=V_{\mathrm{F}}^{-}(j)=0$ for $j \leq j^{*}$, it follows that $j^{*}$ is in fact the smallest $j$ such that $V_{\mathrm{H}}^{-}(j) \geq C$. It will now be shown that $j^{*}=j_{b}$. There are again two subcases.

Subcase $\operatorname{III}(a):\left|E^{+}\right| \geq C(1-p)$. In this case

$$
V_{\mathrm{H}}^{+}(j) \leq V_{\mathrm{F}}^{+}(j) \text { for all } j .
$$

This follows by a routine induction argument. In particular, $V_{\mathrm{H}}^{+}(1)=E^{+} \leq 0=V_{\mathrm{F}}^{+}(1)$. If $V_{\mathrm{H}}^{+}(j) \leq V_{\mathrm{F}}^{+}(j)$ for some $j$ then, since $V_{\mathrm{H}}^{-}(j) \geq V_{\mathrm{F}}^{-}(j)$,

$$
\begin{aligned}
& V_{\mathrm{H}}^{+}(j+1)=p V_{\mathrm{F}}^{+}(j)+(1-p) V_{\mathrm{H}}^{-}(j)+E^{+}, \\
& V_{\mathrm{F}}^{+}(j+1) \geq p V_{\mathrm{F}}^{+}(j)+(1-p)\left\{V_{\mathrm{H}}^{-}(j)-C\right\},
\end{aligned}
$$

so that

$$
V_{\mathrm{H}}^{+}(j+1)-V_{\mathrm{F}}^{+}(j+1) \leq E^{+}+(1-p) C \leq 0 .
$$

By (17), it is always optimal to sell after an up-step. Hence, part (iv) of the optimal strategy follows for the case in which $\left|E^{+}\right| \geq C(1-p)$.

Next, (17) implies that, for $k<j^{*}$,

$$
V_{\mathrm{H}}^{-}(k+1)=E^{-}+(1-q) V_{\mathrm{F}}^{+}(k)+q V_{\mathrm{H}}^{-}(k)=E^{-}+q V_{\mathrm{H}}^{-}(k) .
$$


Hence,

$$
V_{\mathrm{H}}^{-}(j)=\frac{1-q^{j}}{1-q} E^{-}, \quad 1 \leq j \leq j^{*},
$$

and it follows that

$$
j^{*}=\inf \left\{j: \frac{1-q^{j}}{1-q} E^{-} \geq C\right\} .
$$

Since $\left|E^{+}\right| \geq C(1-p),(15)$ and (21) imply that $j^{*} \leq j_{s}$. Hence, by (16) and the definition of $f^{-}(j), j^{*}=j_{b}$. This establishes part (iii) of the optimal strategy for the case in which $\left|E^{+}\right| \geq C(1-p)$.

Subcase III(b): $\left|E^{+}\right|<C(1-p)$. If $j \geq j^{*}$, then equality holds in (18), so that

$$
V_{\mathrm{H}}^{+}(j+1)-V_{\mathrm{F}}^{+}(j+1) \geq E^{+}+C(1-p)>0 .
$$

Thus, if the commodity is being held after an up-step with more than $j^{*}$ time periods remaining, it is optimal to hold it for at least one more time period.

Next, let $j<j^{*}$ be fixed, and suppose that $V_{\mathrm{H}}^{+}(k) \leq 0$ for $k=1, \ldots, j$. Then

$$
V_{\mathrm{H}}^{+}(j+1)=E^{+}+(1-p) V_{\mathrm{H}}^{-}(j) .
$$

Since $j<j^{*}$, (19) holds for $k=0,1, \ldots, j$. Hence, $V_{\mathrm{H}}^{-}(j)$ is given by (20), so the following equivalences hold:

$$
V_{\mathrm{H}}^{+}(j+1) \geq 0 \quad \Longleftrightarrow \quad V_{\mathrm{H}}^{-}(j) \geq \frac{\left|E^{+}\right|}{1-p} \Longleftrightarrow j \geq j_{s} .
$$

If $j^{*} \leq j_{s}$ then (23) implies that $V_{\mathrm{H}}^{+}(j)<0$ for all $j \leq j^{*}$, which means that (20) holds for all $j \leq j^{*}$. Hence,

$$
j^{*}=\inf \left\{j: \frac{1-q^{j}}{1-q} E^{-} \geq C\right\}=j_{b} .
$$

If $j^{*}>j_{s}$ then (20) holds for $1 \leq j \leq j_{s}+1$. However, (23) shows that with $j_{s}+1$ or more time periods remaining, it is no longer optimal to sell after an up-step, so that the calculation of $V_{\mathrm{H}}^{-}(j)$ is different when $j>j_{s}+1$. In fact, for $j_{s}<j<j^{*}, V_{\mathrm{H}}^{+}(j)$ and $V_{\mathrm{H}}^{-}(j)$ satisfy the difference equations (10) and (11), but with different initial conditions. Specifically,

$$
V_{\mathrm{H}}^{-}\left(j_{s}+1\right)=\frac{1-q^{j_{s}+1}}{1-q} E^{-} \quad(\text { by }(20))
$$

and

$$
\begin{aligned}
V_{\mathrm{H}}^{+}\left(j_{s}+1\right) & =E^{+}+p V_{\mathrm{F}}^{+}\left(j_{s}\right)+(1-p) V_{\mathrm{H}}^{-}\left(j_{s}\right) \\
& =E^{+}+(1-p) \frac{1-q^{j_{s}}}{1-q} E^{-}
\end{aligned}
$$

(again by (20) and the fact that $V_{\mathrm{F}}^{+}\left(j_{s}\right)=0$, since $j_{s}<j^{*}$ ). Define

$$
D(k):=D_{\mathrm{H}}^{+}\left(k+j_{s}+1\right), \quad k=0,1,2, \ldots
$$


Then $D(k)=r D(k-1)+\left(E^{+}-E^{-}\right)$for $k=1,2, \ldots, j^{*}-j_{s}-1$ and, hence,

$$
D(k)=r^{k} D(0)+\left(E^{+}-E^{-}\right) \frac{1-r^{k}}{1-r}, \quad k=1,2, \ldots, j^{*}-j_{s}-1,
$$

where

$$
\begin{aligned}
D(0) & =V_{\mathrm{H}}^{+}\left(j_{s}+1\right)-V_{\mathrm{H}}^{-}\left(j_{s}+1\right) \\
& =E^{+}+(1-p) \frac{1-q^{j_{s}}}{1-q} E^{-}-\frac{1-q^{j_{s}+1}}{1-q} E^{-} \\
& =E^{+}+\frac{r q^{j_{s}}-p}{1-q} E^{-} .
\end{aligned}
$$

Now substituting $V_{\mathrm{H}}^{+}(j)=V_{\mathrm{H}}^{-}(j)+D\left(j-j_{s}-1\right)$ into (11) gives

$$
V_{\mathrm{H}}^{-}(j+1)=V_{\mathrm{H}}^{-}(j)+(1-q) D\left(j-j_{s}-1\right)+E^{-}, \quad j=j_{s}+1, \ldots, j^{*}-1 .
$$

Iterating this recursive relationship yields, for $j=j_{s}+2, \ldots, j^{*}$,

$$
V_{\mathrm{H}}^{-}(j)=V_{\mathrm{H}}^{-}\left(j_{s}+1\right)+\sum_{k=1}^{j-j_{s}-1}\left(E^{-}+(1-q) D(k-1)\right) .
$$

After straightforward calculations using (24), (25), and the definitions of $r$ and $\delta$, (26) reduces to

$$
V_{\mathrm{H}}^{-}(j)=\left(j-j_{s}-1\right) \delta+r\left(q^{j_{s}} E^{-}-\delta\right) \frac{1-r^{j-j_{s}-1}}{1-r}+\frac{1-q^{j_{s}+1}}{1-q} E^{-} .
$$

Thus, for all $j \leq j^{*}, V_{\mathrm{H}}^{-}(j)=f^{-}(j)$, and it follows that $j^{*}=j_{b}$, which proves part (iii) of the optimal strategy. Part (iv) follows from the comment following (22) and the equivalences (23) (which hold for $j<j^{*}$ ).

This completes the proof.

Case IV: $E^{+}>0$ and $E^{-} \leq 0$. This case is a mirror image of case III, and can be treated in the same way. The optimal strategy is stated formally in Theorem 3, below.

Analogous to case III, define

$$
j_{s}:=\inf \left\{j \geq 1: 1-p^{j} \geq \frac{(1-p)\left|E^{-}\right|}{(1-q) E^{+}}\right\} .
$$

Let

$$
f^{+}(j):= \begin{cases}\frac{1-p^{j}}{1-p} E^{-}, & j \leq j_{s}+1, \\ \left(j-j_{s}-1\right) \delta+r\left(p^{j_{s}} E^{+}-\delta\right) \frac{1-r^{j-j_{s}-1}}{1-r}+\frac{1-p^{j_{s}+1}}{1-p} E^{+}, & j>j_{s}+1,\end{cases}
$$

and define

$$
j_{b}:=\inf \left\{j \geq 1: f^{+}(j) \geq C\right\}
$$


Theorem 3. If $E^{+}>0$ and $E^{-} \leq 0$ then the optimal strategy is

(i) never sell after an up-step;

(ii) never buy after a down-step;

(iii) buy after an up-step if and only if there are at least $j_{b}$ time periods remaining;

(iv) sell after a down-step if and only if $\left|E^{-}\right| \geq C(1-q)$ or the number of remaining time periods is at most $\min \left(j_{b}, j_{s}\right)$.

\section{Examples of return variance}

In this section we present simulation results to illustrate the variance in return possible when applying optimal trading strategies. Some questions for further study are also mentioned after the simulation results. Simulations are based on data from a mutual fund that tracks the common stock performance of the 1000 largest publicly traded U.S. companies-the Schwab 1000 index fund (SNXFX). The intention is not to advocate that this fund or any commodity necessarily obeys a random walk with correlation. Rather, the example provides motivation for exploring what sort of return variance could be expected if the trading strategies were applied under the RWC assumption. Since the exact distribution on return is difficult to compute even for simple distributions of $Y_{u}$ and $Y_{d}$, simulation is used in the examples given here.

The RWC model used in the simulations is based on the daily closing price of the SNXFX fund from December 1, 2006 to November 30, 2007. Over that time period, after a decrease in share price, the change in share price the next day averaged 0.087 with a standard deviation of 0.421. After an increase in share price, the change in share price the next day averaged -0.048 with a standard deviation of 0.396 . The distributions of price change following a decrease or an increase in share price are unimodal and reasonably approximated by a normal distribution. In the simulation it was assumed that for any trade-buying or selling-1000 shares were traded. So, motivated by the data, an RWC model with $Y_{u} \sim \mathcal{N}(-48,396), p=0.452$, $Y_{d} \sim \mathcal{N}(87,421)$, and $q=0.418$ was used. Here, $\mathcal{N}(\mu, \sigma)$ denotes a normal distribution with mean $\mu$ and standard deviation $\sigma$.

The optimal strategy given by Theorem 2 was applied over a time horizon of $N=60$. For the assumed distributions on $Y_{u}$ and $Y_{d}$, the optimal strategy will buy after a down-step and sell after an up-step whenever the transaction costs, $C$, are below 87. For $C<87, j_{b}=2$ and $j_{s}=1$. The buy-and-hold strategy buys 1000 shares of the fund on the first day and holds them until they are sold at the time horizon. Simulations (100000 Monte Carlo points and the walk assumed to have taken a down-step at time 0) were performed for several values of $C<87$. For $C=0$, the average return for the buy-and-hold strategy was 1114 with a standard deviation of 2817, while the average return for the optimal strategy was 2462 with a standard deviation of 2094. Moreover, for this case, the optimal strategy outperformed the buy-and-hold strategy more than $70 \%$ of the time. The distribution of return for the optimal strategy is shown in Figure 1 for $C=0$. While the optimal strategy significantly improves upon the average return observed for the buy-and-hold strategy, both strategies have fairly large deviations in return. For $C=10$, the average return for the optimal strategy was 2308 with a standard deviation of 2074; for $C=20$, the average return was 2126 with a standard deviation of 2072; for $C=40$, the average return was 1794 with a standard deviation of 2051; and, for $C=80$, the average return was 1149 with a standard deviation of 2020. The examples indicate that, as transaction costs increase, the average performances of the buy-and-hold and optimal strategies become similar. Moreover, the buy-and-hold strategy becomes nearly as likely to outperform 


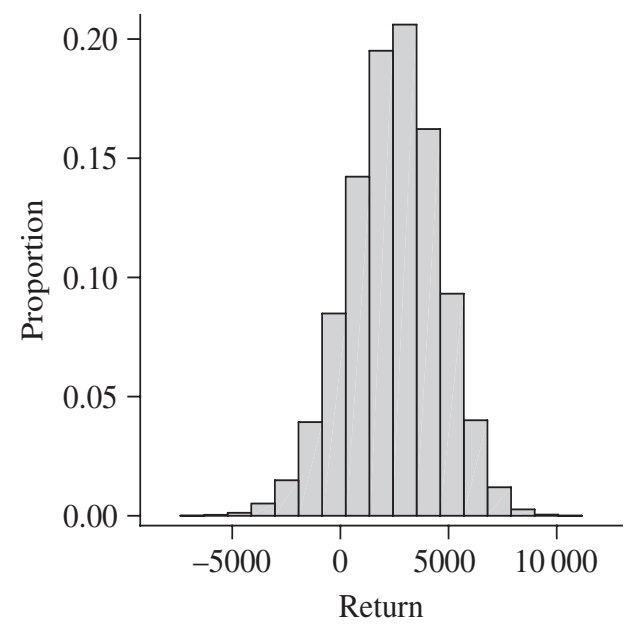

FiguRE 1: Distribution of return for the optimal strategy for $C=0$ and $N=60$ (100000 Monte Carlo points). The mean return was 2462 with a standard deviation of 2094.

the optimal strategy as the optimal strategy is to outperform the buy-and-hold strategy-the buy-and-hold strategy outperforms the optimal strategy $47 \%$ of the time for $C=80$.

It is a bit surprising that the standard deviation in return was lower for the more aggressive optimal strategies than for the buy-and-hold strategies, for all the cases considered. It would be interesting to determine if it is true in general that the variance in return of the optimal strategy is smaller than for the buy-and-hold strategy. Some other natural questions to pursue include the following.

- Many commodity funds place a limit on the number of trades that can occur within a given time. What are optimal strategies given a bound on the number of trades?

- How does a (tax) penalty for rapid buying and selling affect the form of the optimal trading strategy?

- What is the optimal strategy if there is a (dividend) reward for holding the commodity at certain times?

- The optimal strategies given here assume that the same number of units of the commodity are bought and sold each time. What is the optimal strategy when the number of units traded is allowed to vary?

\section{References}

Allaart, P. C. (2004). Optimal stopping rules for correlated random walks with a discount. J. Appl. Prob. 41, $483-496$. Allaart, P. C. And Monticino, M. G. (2001). Optimal stopping rules for directionally reinforced processes. Adv. Appl. Prob. 33, 483-504.

Böнm, W. (2000). The correlated random walk with boundaries: a combinatorial solution. J. Appl. Prob. 37, 470-479.

Chen, A. Y. And Renshaw, E. (1994). The general correlated random walk. J. Appl. Prob. 31, 869-884.

Gillis, J. (1955). Correlated random walk. Proc. Camb. Philos. Soc. 51, 639-651.

GoldsteIn, S. (1951). On diffusion by discontinuous movements, and on the telegraph equation. Quart. J. Mech. 4, $129-156$

Henderson, R. And Renshaw, E. (1980). Spatial stochastic models and computer simulation applied to the study of tree root systems. Compstat 80, 389-395.

Iossif, G. (1986). Return probabilities for correlated random walks. J. Appl. Prob. 23, 201-207. 
JAIN, G. C. (1971). Some results in a correlated random walk. Canad. Math. Bull. 14, 341-347.

JAIN, G. C. (1973). On the expected number of visits of a particle before absorption in a correlated random walk. Canad. Math. Bull. 16, 389-395.

Malkiel, B. G. (1999). A Random Walk Down Wall Street, 7th edn. Norton, New York.

Mauldin, R. D., Monticino, M. G. and Von Weizsäcker, H. (1996). Directionally reinforced random walks. Adv. Math. 117, 239-252.

MoHan, C. (1955). The gambler's ruin problem with correlation. Biometrika 42, 486-493.

Mukherjea, A. And Steele, D. (1986). Conditional expected durations of play given the ultimate outcome for a correlated random walk. Statist. Prob. Lett. 4, 237-243.

MukherJeA, A. AND STEele, D. (1987). Occupation probability of a correlated random walk and a correlated ruin problem. Statist. Prob. Lett. 5, 105-111.

Proudfoot, A. D. and Lampard, D. G. (1972). A random walk problem with correlation. J. Appl. Prob. 9, $436-440$.

Renshaw, E. And Henderson, R. (1981). The correlated random walk. J. Appl. Prob. 18, 403-414.

SETH, A. (1963). The correlated unrestricted random walk. J. R. Statist. Soc. B 25, 394-400.

ZHANG, Y. L. (1992). Some problems on a one-dimensional correlated random walk with various types of barrier. J. Appl. Prob. 29, 196-201. 